\title{
LA AGRICULTURA EN LA MONTAÑA DE ALICANTE: SITUACIÓN ACTUAL Y POTENCIAL FUTURO
}

\author{
María Hernández Hernández \\ Enrique Moltó Mantero
}

\section{RESUMEN}

Las actuaciones diversificadoras de las actividades económicas en el medio rural presentan una marcada orientación hacia las actividades relacionadas con el turismo de interior. Sin embargo, el potenciamiento de las actividades rurales mediante la mejora de los cultivos tradicionales, la difusión de nuevos aprovechamientos o la introducción de mejoras técnicas (bancos de tierras y de servicios) o la propia pluriactividad agraria puede contribuir a su mantenimiento. Las iniciativas deben abogar por una defensa de la calidad, de la originalidad de estos productos frente a la cantidad que ofrecen las áreas donde la intensificación es más factible. El mantenimiento de las actividades agrarias facilita, asimismo, la conservación de unos paisajes cuyo abandono genera notables problemas de degradación ambiental. Sin embargo, el problema reside en convertir las externalidades que ofrecen estos paisajes antropogénicos en un «bien de mercado».

Palabras clave: políticas estructurales, diversificación de rentas, comercialización, denominaciones de origen, pluriactividad, Ceder Aitana, cooperativas.

\begin{abstract}
The diversifying proceedings of economic activities on the rural areas show a remarkable guidance with activities connected with rural tourism. Nevertheless, the development of rural activities by the improvement of traditional crops and the spreading of new ones, the introduction of new crop methods and the incomes generated by having more than one job can help to keep them. These enterprises have to defend the originality of these products and its quality in charge of its quantity. Supporting these farming activities allow, moreover, the preservation of landscapes, whose giving up produce noteworthy problems on the environment. The issue is to transform the «octward goods» that offer these anthropogenic landscapes in a «market goog».
\end{abstract}

Key words: structural policies, income diversification, marketing, prestigious product classification, label, ceder Aitana, cooperatives. 


\section{Introducción}

El ámbito geográfico objeto de estudio se sitúa en el nordeste de la provincia de Alicante, englobando 46 municipios de las comarcas del Alcoià, del Comtat, l'Alacantí y las Marinas. Espacio que recibe la significativa calificación de «La Montaña»; adjetivo que refleja las características fisiográficas predominantes en esta área supracomarcal. Dicho territorio ha sido conocido tradicionalmente por su desarrollo urbano-industrial centrado fundamentalmente en el eje Alcoy-Cocentaina-Muro (PÉREZ PÉREZ, D.; 1997), que concentra alrededor del $80 \%$ de la población de este espacio, quedando el resto repartido entre 44 núcleos rurales. Es necesario precisar que Alcoy, con más de 60.000 habitantes, por sus características demográficas y socioeconómicas, queda excluido del CEDER Aitana, aunque su influencia en esta área de actuación no puede ser ignorada. Se llegó, también, a discutir la inclusión de los otros dos municipios del eje anteriormente enunciado, Cocentaina y Muro, casi conurbados con Alcoy (RODRIGO ALFONSO, C.; 1999), en un programa de desarrollo rural como el LEADER, más aún que por su peso demográfico, suman entre los dos más de 17.000 habitantes, por la actividad económica predominante en los mismos, la industria.

Menos discutible resulta la inserción de Callosa d'en Sarrià, a pesar de sus más de 6.000 habitantes, porque la actividad predominante en este municipio es la agrícola. No obstante, la agricultura de Callosa, altamente intensificada y competitiva, hasta el punto de permitir en buena medida una plena dedicación a los titulares de las explotaciones, tiene poco que ver con la predominante en buena parte de los municipios del CEDER, tradicionalmente dedicada al autoconsumo, generalmente extensiva y de secano, con rentabilidades que difícilmente hacen que en la actualidad se constituya como única o principal fuente de ingreso de la renta familiar, y cuya supervivencia se entiende, sobre todo, gracias a su papel como complemento de otra actividad económica principal, y, en el caso de pequeñas parcelas, como simple recreo de fin de semana o vacacional para los residentes en ciudades próximas (MOLTÓ MANTERO, E.; 1998).

La heterogeneidad de este espacio geográfico, marcada esencialmente por la dualidad entre los focos urbano-industriales y los rurales, es aun mayor si tenemos en cuenta que tampoco encontramos ninguna uniformidad dentro de los propios núcleos rurales desde el punto de vista territorial, económico y demográfico. Algunos de estos pequeños municipios rurales, como Beniarrés, Benilloba o Alquería de Aznar, gozan de una clara tradición industrial, fruto en buena medida de la influencia del eje anteriormente mencionado, mientras otros siguen teniendo un predominio relativo de la actividad agraria. Pasamos de densidades muy bajas con poblaciones absolutas de menos de 100 habitantes como Tollos (42 habitantes) a densidades relativamente elevadas y poblaciones superiores a los 1.000 habitantes como Beniarrés (1.503 habitantes). Significativa resulta asimismo la coexistencia entre las denominadas en la zona «grandes explotaciones» y las pequeñas y medianas.

Un breve comentario requiere, asimismo, la catalogación de este espacio geográfico como área deprimida, pero podríamos preguntarnos «deprimido con respecto a qué». Hemos de aceptar que dentro del contexto de una provincia como la de Alicante, caracterizada por un dinamismo económico y demográfico relevante, un sector interior con predominio de las actividades agrarias y que ha experimentado unas pérdidas demográficas destacables desde los años cincuenta, aceptaría tal calificativo. Sin embargo, si lo comparamos con otros medios rurales interiores, este comarca se constituiría como un espacio privilegiado. Dada su posición se beneficia de la proximidad a núcleos urbanos de tamaño medio-grande, de los ejes industriales interiores y del dinamismo del sector litoral (servicios y turismo). Esto ha permitido que, frente a otras áreas, donde ha predominado el 
abandono total de los pueblos, aquí se practique el pendularismo. Este movimiento se ve favorecido por la citada proximidad geográfica y genera notables beneficios económicos (conservación de tierras cultivadas, pluriactividad, etc.) y sociales (centros educativos, centros de salud, ocio, etc.). En definitiva, este espacio cuenta con los atractivos de un área rural (calidad de vida), pero sin los inconvenientes de un rural profundo.

\section{Actuaciones en sectores agrícolas consolidados}

Estas intervenciones están generalmente dirigidas a las cooperativas en las que estos sectores ya hace bastante tiempo que están organizados por razones evidentes de comodidad y eficacia, ya que se constituyen generalmente como las grandes dinamizadoras de sus respectivas producciones. Las inversiones, beneficiadas con subvenciones, se orientan generalmente a la renovación de la maquinaría con la que se procesan y comercializan todos estos productos y a la reforma de los locales que albergan estas máquinas. No debemos, sin embargo, olvidar las iniciativas llevadas a cabo estrictamente desde el ámbito privado, sin un apoyo asegurado en principio por parte de las administraciones públicas. Es conveniente apuntar, asimismo, que el sector oleícola es el más dinámico a la hora de solicitar proyectos, hecho que no deja de ser lógico dada la gran relevancia del aceite de oliva en la economía agraria de la Montaña. (cuadro 1)

Cuadro 1

SUPERFICIES OCUPADAS POR LOS PRINCIPALES CULTIVOS DE LA MONTAÑA DE ALICANTE (1998)

\begin{tabular}{|l|c|c|c|c|c|c|c|c|c|c|}
\hline \multirow{2}{*}{} & \multicolumn{4}{|c|}{ Olivo } & \multicolumn{4}{c|}{ cerezo } & \multicolumn{3}{c|}{ níspero } \\
\cline { 2 - 12 } & \multicolumn{2}{|c|}{ secano } & \multicolumn{2}{c|}{ regadín } & \multicolumn{2}{c|}{ secano } & \multicolumn{2}{c|}{ regadío } & \multicolumn{2}{c|}{ regadío } \\
\cline { 2 - 12 } & has & $\%$ & has & $\%$ & has & $\%$ & has & $\%$ & has & $\%$ \\
\hline prov. Alic. & 30855 & - & 3257 & - & 1435 & - & 244 & - & 1495 & - \\
\hline Montaña Alic. & 13827 & 44,8 & 455 & 13,9 & 1259 & 87,7 & 1 & 0,4 & 764 & 51,1 \\
\hline
\end{tabular}

FUENTE: Hojas 1-T. Elaboración propia.

\subsection{Actuaciones en el sector olivarero}

En el olivo, principal aprovechamiento de la comarca (cuadro 1), existe un especial interés en la introducción de la molturación en dos fases en las distintas almazaras de esta comarca y en el correcto tratamiento del alpechín, con el fin de evitar los problemas ecológicos derivados de su vertido a los cursos fluviales. Se camina también hacia una mejora de los equipamientos y las infraestructuras y hacia una renovación de toda la maquinaria que exige el tratamiento de este producto, más allá de la molturación y el tratamiento del alpechín anteriormente enunciados, como por ejemplo la destinada a la limpieza de la aceituna o al correcto almacenamiento del aceite.

En el apartado referido a las cooperativas podemos citar varias iniciativas del sector olivarero en distintas fases de la producción: la elaboración del aceite con la construcción de la nueva almazara de la Cooperativa Agrícola Católica de Cocentaina, la comercialización a nivel de toda la Comunidad Valenciana con la creación de una Agrupación de Intereses Económicos (A.I.E.) y el tratamiento de los subproductos de la elaboración del aceite, alpeorujo y alpechín, con la constitución de una Cooperativa de Segundo Grado en 
Cuatretonda formada por diversas cooperativas del sur de la provincia de Valencia y el norte de la de Alicante.

En las actuaciones privadas cabe citar dos tipos de actuaciones completamente contrapuestas, adaptadas cada una de ellas a la heterogeneidad propia de esta comarca, y capaces de coexistir, debidamente delimitadas, en la realidad plural de este espacio geográfico. Por un lado, de las entrevistas realizadas a algunos de los escasos agricultores a título principal en la comarca, se deduce un intento por intensificar la producción en sectores tradicionales como el olivo o el almendro, en aquellas parcelas donde esto es materialmente posible, con nuevos marcos de plantación más reducidos y nuevas variedades especialmente productivas, todo ello adaptado a la introducción de maquinaria en todas las fases de producción de estos cultivos, incluida una especialmente gravosa por el elevado coste de la mano de obra, la recolección. En el contexto de esta intensificación no se descarta la progresiva extensión del riego localizado, necesario en años excesivamente secos para reducir la vecería, especialmente del olivo, y también exigido por marcos de plantación más pequeños que los tradicionales. En el polo opuesto a estas actuaciones cabe citar la difusión del cultivo del olivo ecológico, que se analizará en el apartado relativo a la adopción de nuevos sistemas de cultivo (epígrafe 3.3).

\subsubsection{La remodelación de las instalaciones}

La Cooperativa Agrícola Católica de Cocentaina abordó la necesaria construcción de una nueva almazara con los modernos sistemas de extracción del aceite en dos fases, una mayor y mejor capacidad de almacenamiento, sistema automático de pesado, limpieza de

Cuadro 2

\section{INVERSIONES DEL CEDER AITANA}

\begin{tabular}{|l|l|c|r|r|}
\hline Sector & & $\mathbf{N}^{\circ}$ solicitudes & inversión & Subvención \\
\hline Oleícola & Mejora maquinaria & 7 & 89.640 .000 & 23.735 .127 \\
& Acond. Instalaciones & 6 & 69.808 .259 & 19.614 .484 \\
& Prod. Ecológica & 2 & 81.925 .500 & 18.239 .354 \\
\hline Cerezas & Mejora comercial. & 1 & 5.998 .900 & 2.339 .560 \\
& equipo informático & 1 & 5.505 .610 & 2.202 .244 \\
\hline Frutos secos & Mejora maquinaria & 1 & 12.952 .007 & 3.885 .602 \\
\hline Granjas & Avestruces (instalación) & 1 & 5.244 .819 & 1.573 .446 \\
& Avícolas & 1 & 27.642 .000 & 5.528 .400 \\
\hline Frutales trop. & Plantación & 1 & 11.741 .512 & 4.696 .606 \\
\hline Floricultura & Instalación & 2 & 8.116 .000 & 3.246 .400 \\
\hline Ind. Agroalim. & acond. Instalaciones & 1 & 3.000 .000 & 900.000 \\
\hline \multicolumn{2}{|r|}{ Total línea B5 } & 25 & 329.884 .864 & 88.346 .223 \\
\hline \multicolumn{2}{|r|}{ Total programa } & 102 & 1.240 .042 .400 & 463.976 .494 \\
\hline
\end{tabular}

FUENTE: CEDER Aitana. Datos a 30-VI-1999. Elaboración propia. 
la aceituna, labor antes realizada por cada productor, y un más cómodo método de descarga para el socio. Aunque se obtuvieron algunas subvenciones, menos de las prometidas en un principio, entre ellas una del propio LEADER II (cuadro 2) sólo para la estricta fase de extracción del aceite, casi toda la inversión fue sufragada por los socios mediante la retención de un $25 \%$ de su subvención a la producción durante un número determinado de años, cinco en principio, más tarde ocho. Mención aparte merecería la posible desproporción entre la gran capacidad de molturación de la almazara y el tope máximo de producción, claramente más bajo, el fallo de algunas de las prometidas subvenciones y el desfase de la inversión inicialmente presupuestada, de prácticamente un $100 \%$, pero quedaría demostrado en este caso la utilización de las subvenciones para aquello para lo que siempre estuvieron pensadas, una mejora de la producción. Cabe citar en este punto, por la incidencia que pueda tener en esta inversión aún sin cubrir, el grave perjuicio ocasionado por el cambio en la política de subvenciones de la nueva OCM del aceite en una zona donde predominan los pequeños propietarios y donde la productividad media de los olivos es mucho más baja que la de las grandes zonas olivareras españolas. Se demuestra de nuevo que la aplicación de políticas homogéneas a realidades plurales es mucho más cómoda para las administraciones públicas, pero crea graves agravios productivos entre cada una de estas realidades.

\subsubsection{El tratamiento del alpechín}

Las exigencias de un correcto tratamiento que evite los vertidos y la contaminación de los cursos fluviales, especialmente allí donde se estancan, charcas y embalses, ha creado graves problemas a todas las almazaras, acostumbradas a verter los alpechines sin ningún tipo de control, y las de esta comarca no han sido una excepción. Varias han sido las posibles soluciones adoptadas. La cooperativa de $2^{\circ}$ grado de SOCAPMA presentó un proyecto, especialmente concreto y fundamentado, que fue apoyado por el LEADER II con 14.789.354 pesetas sobre un total presupuestado de 70.425.500 (cuadro 2).

En otras ocasiones, las almazaras intentan solucionar sus problemas con la participación en empresas orujeras como Orujos de Levante en Yecla (Murcia), en las que a cambio de sus inversiones pueden transportar sus alpechines, sin ninguna seguridad sobre la rentabilidad de las mismas, llegando, según los años y la evolución de los precios del aceite de orujo, a no sólo a no percibir dinero de estos subproductos, sino a tener que pagar el transporte del mismo. En algún caso se apuesta también por aprovechar este alpechín como fertilizante, en un auténtico ejemplo de agricultura integrada, pero los resultados no están garantizados y puede ser necesaria una mayor manipulación de este subproducto para su correcta utilización como fertilizante.

La última propuesta es la instalación de una planta integrada en la que se extraigan todos y cada uno de los subproductos del alpechín (orujo, fertilizantes, etc.) y se comercialicen de manera que permitan como mínimo cubrir los costes de construcción y mantenimiento de esta planta así como el transporte del alpechín hasta la misma por parte de todas y cada una de las distintas almazaras. Su creación y posterior funcionamiento precisa, no obstante, de una serie de premisas previas. En primer lugar, se debía buscar una planta que tuviera una ubicación lo suficientemente central como para permitir el acceso más o menos próximo a la misma por parte de un buen número de almazaras, de manera que la construcción y mantenimiento de las instalaciones sea rentable. También resulta conveniente la preexistencia de unas instalaciones apropiadas para asumir este tipo de manipulaciones sin demasiadas adaptaciones. Es necesario igualmente el apoyo claro de la administración autonómica al proyecto, tanto de la Conselleria de Agricultura como de la de Medio 


\section{Cuadro 3}

APORTACIONES AL CAPITAL SOCIAL DE LA COOPERATIVA DE $2^{\circ}$ GRADO DE QUATRETONDETA

\begin{tabular}{|lr|lr|}
\hline Cooperativas & Aportac. capital social & Cooperativas & Aportac. capital social \\
\hline & & & \\
Ayelo Malferit & 300.000 & Jarafuel & 3.500 .000 \\
Alcublas & 1.500 .000 & Liria & 2.350 .000 \\
Beniarrés & 2.200 .000 & Mojente & 3.000 .000 \\
Benimantell & 750.000 & Muro Alcoy (Socapma) & 12.000 .000 \\
Biar & 2.350 .000 & Navarrés & 4.000 .000 \\
Buñol & 750.000 & Pedralva & 500.000 \\
Chelva & 1.200 .000 & Quatretonda & 4.000 .000 \\
Chiva & 1.750 .000 & Sax & 2.200 .000 \\
Chulilla & 600.000 & Tárbena & 500.000 \\
Cocentaina & 4.150 .000 & Torremanzanas & 850.000 \\
Cortes de Pallas & 850.000 & Tuejar & 500.000 \\
Gayanes & 1.750 .000 & Turis & 3.250 .000 \\
Gata de Gorgos & 300.000 & Venta del Moro & 1.400 .000 \\
\cline { 2 - 3 } Jalance & 1.300 .000 & TOTAL & $\mathbf{5 7 . 8 5 0 . 0 0 0}$ \\
\hline
\end{tabular}

Módulo para calcular la aportación al capital social: se multiplica el número de olivos por el importe estimado por olivo. Base de cálculo: $<2.500$ olivos x 33 ptas; $<5.000$ olivos x 32 ptas; $<7.500$ olivos x 31 ptas; $<10.000$ ptas x 29 ptas; $<15.000$ olivos x 25 ptas; $<20.000$ olivos x 21 ptas; $<30.000$ olivos x 17 ptas; $>30.000$ olivos x 15 ptas. Fuente: Cooperativa de Cocentaina.

Ambiente. Por todo ello es especialmente interesante que un buen número de las cooperativas del sur de la provincia de Valencia y el norte de la de Alicante (alrededor de una treintena de socios) sean capaces de apoyar un proyecto concreto, que reúne buena parte de las condiciones anteriormente expresadas, con la constitución de una cooperativa de segundo grado con la adaptación de una antigua alcoholera en Cuatretonda para el tratamiento del alpechín. El objetivo primordial de las cooperativas asociadas es el de que los ingresos generados por la venta de los subproductos cubra las inversiones realizadas en la construcción y el mantenimiento de la planta y el transporte hasta la misma, tal y como confirma el análisis de la aportación de cada una de ellas al capital social de esta entidad (cuadro 3), resultando de momento objetivo secundario y a más largo plazo la posibilidad de conseguir algún tipo de beneficio extra.

\subsubsection{Políticas de valorización de la producción}

Estas políticas tienen como objetivo fundamental mejorar la calidad del aceite, un punto donde sí puede ser muy competitivo este espacio productivo, para el que se podría y se debería buscar una denominación de origen, ya que, en buena parte de estas explotaciones, dadas las características orográficas predominantes, el parcelario y el tamaño medio de las explotaciones, en líneas generales resulta difícil intensificar la producción.

A nivel de toda la Comunidad Valenciana, en el campo de la comercialización, podemos citar la reciente creación de una sociedad mercantil (A.I.E.), que agrupa a las dos grandes marcas bajo las que comercializan su aceite de oliva virgen casi todas las coopera- 
tivas olivareras de esta comunidad autónoma, Oli d'Or y Olival. Ninguna de las dos grandes marcas desaparecería, quedando Olival para la distribución a los socios y almacenes de venta de todas las almazaras integradas en esta agrupación, y Oli d'Or, más conocido para el consumidor de la Comunidad Valenciana, para su distribución en los comercios. Por su parte, se reserva la posibilidad de que determinadas variedades muy valoradas como la del aceite de la aceituna blanqueta, predominante en buena parte de La Montaña, aparezcan como submarca bajo la marca genérica.

Esta asociación, valorada positivamente y hasta cierto punto auspiciada por la Administración autonómica, pretende reducir distintos costes, de entre los que podemos destacar el envasado, la comercialización y el correcto tratamiento de los subproductos de la elaboración del aceite. Otra de las ventajas de esta unión es que como tal sociedad mercantil podría actuar de modo más flexible desde el punto de vista legal y económico donde no puede hacerlo como cooperativa agrícola, como, por ejemplo, en la compra de aceite para asegurarse una cuota de producción en años en los que la cosecha de la Comunidad Valenciana fuese especialmente mala. Se pretende también iniciar desde esta sociedad nuevas líneas de producción con la creación y distribución de nuevos productos de creciente demanda como el aceite de oliva ecológico o los aceites aromáticos, o el aceite en pequeños envases para su utilización en la alta restauración.

Se detecta cierto acercamiento entre las distintas cooperativas de esta comarca, tradicionalmente divididas entre la Cooperativa Agrícola Católica de Cocentaina, segunda en capacidad productiva y que comercializa su producción bajo la marca Oli d'Or, y la Cooperativa de segundo grado SOCAPMA, primera en capacidad productiva y que asocia a más de cuarenta cooperativas, y las dos más pequeñas de Gayanes y Beniarrés, las tres integradas en la marca Olival. Se trataría de que integradas todas en el marco general de la A.I.E. fueran capaces de unirse y hacer valer su peso específico y sus características propias frente a otras cooperativas de la Comunidad Valenciana.

Es pronto para evaluar la efectividad de esta sociedad mercantil, constituida en abril de 1999, y en una fase que podemos considerar inicial, lejos de conseguir los objetivos fijados, pero se pueden detectar problemas por la clásica desconfianza de todas las cooperativas entre sí y frente a esta entidad superior, manejada por técnicos, difícilmente comprendidos por los consejos rectores de las cooperativas, formados básicamente por agricultores de mentalidad esencialmente conservadora. Particularmente desde las cooperativas e incluso desde la propia A.I.E., aunque no se admita vox publica, se sigue prefiriendo la venta a granel de estos aceites de oliva virgen de gran calidad a los corredores, que a su vez lo venderán a las grandes marcas nacionales e internacionales, que lo emplearán para mejorar la calidad de otros aceites o para comercializarlo directamente como aceite de oliva virgen, incluso bajo una denominación de origen de distintos lugares de España. Se cree que es la solución más rápida, práctica y directa, y también la más económica, por ser la que menos gastos de producción conlleva, especialmente al no tener que invertir en el envasado, y menos riesgos ante las posibles fluctuaciones de mercado. Se tendría que tener un gran convencimiento desde los técnicos de la Sociedad Mercantil con un gran poder demostrativo y de convicción, para que los productores entendieran que las inversiones en un envasado de calidad (envases de cristal de capacidad siempre inferior al litro), los gastos exigidos para llevar a cabo una comercialización directa del producto, la apuesta por nuevas líneas de producción como los aceites ecológicos o aromáticos, la creación en su caso de alguna denominación de origen, a largo plazo revertirían en una mayor rentabilidad de sus cultivos. Pero el hecho es que buena parte de los productores valencianos integrados en estas cooperativas son agricultores a título secundario y para ellos, su explotación no es más que un recreo, la obtención de un producto de autoconsumo o, como mucho, un 
complemento de rentas, y, por ello, imbuidos además por su tradicional carácter conservador, muchos de ellos son mayores de cincuenta años y tienen dudas sobre la continuidad de su explotación, son reacios a que se les vendan castillos en el aire, y prefieren no arriesgarse pero obtener un beneficio menor pero seguro.

\subsection{Actuaciones en frutales de hueso (cereza y níspero)}

Las inversiones efectuadas en ambos cultivos presentan diferencias significativas con respecto al olivo dada la menor superficie ocupada pero también las diferentes realidades que representan tal y como sucede con el níspero cuya rentabilidad permite su práctica por agricultores a tiempo completo (cuadro 1).

En el caso del níspero no son posibles substanciales ayudas porque se trata de una cooperativa que supera los 1.300 millones de facturación anual y emplea a más de 50 trabajadores, y, por ello, no es una PYME sino una gran empresa según el baremo LEADER y queda excluida de ayudas directas. No obstante, ha recibido subvenciones una sección destinada a la comercialización. En ella se apoya la creación de nuevos tipos de envasado distinta a los tradicionales como el vidrio o el tetra brick y líneas de producción que exigen cierta manipulación del producto tales como zumos o mermeladas. Estos productos son envasados en Murcia porque resulta más rentable hacerlo así que montar una gran envasadora en el municipio de origen. Actuaciones a las que debemos unir las campañas de difusión de este producto en diversos mercados europeos al amparo de su denominación de origen. A éstas, se unen las numerosas subvenciones amparadas por los reglamentos estructurales y orientadas a la modernización de las estructuras agrarias (instalación de riego por goteo, mejora de variedades, instalación de cortavientos, etc.), que han contribuido a mantener el dinamismo de las actividades agrarias en este espacio.

La cereza registra también una menor receptividad a la hora de presentar proyectos que el sector oleícola, aunque últimamente se han recibido de manera masiva (cuadro 2). Los motivos que justifican esta menor aceptación son diferentes a los del níspero. Una posible causa de esta menor receptividad reside en las diferencias que se perciben entre los productores asociados a las cooperativas y los técnicos del consejo regulador de la denominación de origen. Los primeros tienen planes más realistas mientras que los segundos están más interesados en la adopción de proyectos, a veces faraónicos, que intensifiquen la mecanización de las explotaciones y la mejora del envasado (máquinas de calibres) a pesar de su dudosa aplicación a las producciones de esta zona: se orientan a variedades con escasa difusión en la zona como la burlat, y de poca viabilidad económica dada la baja producción, $1.400 .000 \mathrm{~kg}$. Se detecta un problema sobre el que cabría actuar como es la pérdida de variedades autóctonas, sustituidas por variedades con mayor facilidad para su comercialización. Las actuaciones tienen como objetivo prioritario la mejora de la maquinaria para comercializar el fruto, que se focaliza en la localidad de Patró (Vall de Gallinera). El Ceder ha apoyado también un estudio acerca del valor nutritivo de la cereza y el desarrollo de unas parcelas experimentales para introducción de nuevas variedades y la instalación de equipos informáticos en las oficinas.

\section{Diversificación de los aprovechamientos agrícolas}

La introducción de sectores que generen rentas a los habitantes de estos espacios es uno de los objetivos definitorios de los programas de desarrollo rural. No obstante, se incide generalmente en la introducción de actividades extra-agrarias relacionadas con el turismo de interior, sin considerar que estos medios podrían ofrecer una serie de posibilidades 
relacionadas con los aprovechamientos tradicionales pero también con nuevos usos agrícolas, que permitirán complementar rentas mediante la pluriactividad agraria. Estos últimos se relacionan con la potenciación de aprovechamientos que crecían espontáneamente (aromáticas), la introducción de cultivos novedosos (flores y cultivos tropicales), la difusión de actividades ganaderas (cría de avestruces). Prácticas asociadas generalmente a técnicas no convencionales (agricultura ecológica), que permiten incrementar las rentas obtenidas por unidad de superficie, y junto a éstas las mejoras introducidas en la gestión de la explotación (banco de tierras).

\subsection{El cultivo de aromáticas}

Los cultivos de plantas aromáticas de honda tradición en la comarca, tal como corrobora Cavanilles al recorrer estas tierras en el siglo XVIII, no han sido considerados por sus habitantes como un cultivo, sino más bien hierbas que crecían de manera espontánea en linderos, eriales o en los sectores de monte bajo. Su reciente expansión enlaza con el auge que han experimentado el uso de productos naturales y concretamente la destilación de aceites, el secado de plantas para herboristería, la fabricación de aromas o su empleo en la cocina y en la industria farmacéutica. Esta evolución determina que la comercialización, una de las carencias de la agricultura tradicional, esté asegurada, ya que existe más demanda que producción. Contando ésta además con la ventaja de realizarse a través de una marca (Herbes del Molí) con creciente implantación en el mercado, evitando la venta a granel y su comercialización a través de intermediarios.

No obstante, su difusión se ve dificultada por una serie de carencias; merecen citarse ciertas deficiencias en las técnicas de fabricación y comercialización y, sobre todo, el escaso convencimiento por parte de los agricultores para introducir este cultivo. Aspecto éste que se acentúa por la insuficiente dinamicidad del mercado de la tierra: muchos propietarios no cultivan sus parcelas pero tampoco se muestran favorables a cederlas a otros que eligen esta opción y que precisan ampliar la superficie cultivada. Las actuaciones subvencionadas por el CEDER Aitana se han orientado hacia la atenuación de estas lagunas, al fomentar la formación, la creación de bancos de tierras y la difusión de cultivos ecológicos, ya que aquellos que se practican mediante «técnicas convencionales» quedan excluidos de estas ayudas para evitar el solapamiento con las subvenciones de la Conselleria de Medio Ambiente (HERNÁNDEZ HERNÁNDEZ, M.; en prensa). Las técnicas ecológicas se concentran en un elevado porcentaje en la cooperativa Blancoana (Alfafara), que además de dedicarse a la elaboración de estos productos prevé la visita a ciertas instalaciones, especialmente aquéllas orientadas a la obtención de esencias mediante técnicas tradicionales, obteniendo de este modo ingresos complementarios (venta directa a los potenciales visitantes). Sin embargo, la novedad más significativa se ha producido en el sistema de tenencia de las tierras al crear un banco de tierras que permite a aquellos propietarios que no pueden o no quieren cultivar sus parcelas, cederlas mediante un contrato. Reduciendo de este modo los terrazgos baldíos e incrementando las superficies roturadas; aspecto imprescindible para que un aprovechamiento extensivo sea rentable.

\subsection{Las actividades ganaderas}

La introducción de la ganadería en las comarcas de interior fue una opción de la Conselleria de Agricultura a finales de los setenta para diversificar rentas en estos espacios deprimidos. Centrándose en una primera fase en la cría de aves y cerdos. No obstante, esta actividad a diferencia de lo que ocurrió en otras comarcas interiores valencianas tuvo una 


\section{Cuadro 4}

EVOLUCIÓN SOLICITUDES ACOGIDOS A LAS AYUDAS ESTRUCTURALES

(R.D. 808/87, 1887/91 Y 204/96)

\begin{tabular}{|c|c|c|c|c|c|c|c|c|c|c|}
\hline & \multicolumn{3}{|c|}{ 0. Alcoì̀-Comtat } & \multicolumn{3}{|c|}{ 0. Vega Baja } & \multicolumn{3}{|c|}{ total provincial } \\
\hline & & $\mathrm{n}^{0}$ & Inv* & sub* & $\mathbf{n}^{0}$ & inv* & sub* $^{*}$ & $\mathrm{n}^{0}$ & inv* & sub* \\
\hline \multirow[t]{6}{*}{808} & A & 1 & 5.950 & 934 & 9 & 58.691 & 13.814 & 217 & 1.101 .000 & 521.000 \\
\hline & B & 6 & 17.700 & 6.458 & 181 & 340.130 & 134.700 & 1.003 & 2.053 .000 & 694.000 \\
\hline & C & 1 & - & 155 & 6 & - & 3.563 & 3 & - & 200 \\
\hline & D & - & - & - & 1 & - & 2.336 & 2 & - & 4.000 \\
\hline & E & - & - & - & - & - & - & - & - & - \\
\hline & G & - & - & - & - & - & - & - & - & - \\
\hline \multirow[t]{6}{*}{1887} & A & 9 & 43.550 & 13.252 & 57 & 279.942 & 101.862 & 181 & 1.118 .179 & 441.975 \\
\hline & B & 8 & 23.059 & 9.876 & 43 & 273.084 & 36.579 & 118 & 7.159 .668 & 177.313 \\
\hline & $\mathrm{C}$ & - & - & - & - & - & - & 1 & - & 243 \\
\hline & D & - & - & - & - & - & - & - & - & - \\
\hline & $\mathrm{H}$ & 13 & - & 323 & 13 & - & 505 & 101 & 9.738 & 2.881 \\
\hline & $\mathrm{T}$ & - & - & - & 1 & 3.663 & 431 & 2 & 9.738 & 2.604 \\
\hline \multirow[t]{6}{*}{204} & A & 5 & 30.872 & 13.847 & 33 & 141.780 & 81.681 & 120 & 724.220 & 309.420 \\
\hline & B & 3 & 13.826 & 1.996 & 41 & 379.320 & 127.220 & 133 & 771.010 & 317.470 \\
\hline & C & 1 & - & 180 & 14 & - & 3.416 & 15 & - & 3.512 \\
\hline & D & - & - & - & 1 & 16.665 & 8.920 & 1 & 16.665 & 8.920 \\
\hline & $\mathrm{H}$ & 35 & - & 1.048 & 32 & - & 1.058 & 193 & - & 7.441 \\
\hline & $\mathrm{T}$ & 1 & 750 & 62 & 1 & 7.800 & 1.510 & 3 & 10.750 & 5.172 \\
\hline
\end{tabular}

Líneas de actuación: A: primera instalación; B: inversiones en planes de mejora; C: introducción de la contabilidad; D: ayudas a agrupaciones de agricultores; E: zonas de montaña desfavorecida; G: ayudas a la introducción de medidas forestales y actividades turísticas en explotaciones agrarias; H: formación; T: compra de tierras. * por mil; incluida subvención y bonificación de intereses. Fuente: Conselleria de Agricultura. Elaboración propia.

escasa difusión en la Montaña de Alicante y en general en la provincia de Alicante ${ }^{1}$, ya que en ésta el sector manufacturero proporcionaba rentas suficientes; además su práctica requería una dedicación casi exclusiva, lo que la convertía en una práctica poco atractiva como complemento de rentas.

Una mayor aceptación ha tenido la creación de granjas de avestruces o la mejora de la ganadería tradicional (ovejas y cabras), que han recibido ayudas procedentes del Ceder Aitana y de los reglamentos estructurales (R.D. 808/87, 1887/91 y 204/96); aspecto posibilitado por la coincidencia de actuaciones que pueden ser subvencionadas por ambas actuaciones: actividad innovadora y mejora de las estructuras, respectivamente. No obstante, en ocasiones se producen conflictos competenciales, ya que ciertas peticiones no pueden ser acogidas por el Ceder al no ser innovadoras, pero son rechazadas por la Conselleria al no adaptarse a los principios definidores de este decreto (HERNÁNDEZ HERNÁNDEZ, M.;

1 La ganadería aporta un $20 \%$ a la Producción Final Agraria de la C. Valenciana. Sin embargo, las actividades ganaderas tienen un mayor peso específico en Castellón (37\%) que en Valencia (18\%) y Alicante $(7 \%)$. 
en prensa). Estos reglamentos priman a los agricultores a tiempo completo, lo que excluye consiguientemente a la mayor parte de los activos agrarios de este espacio; aspecto que justifica las escasas solicitudes acogidas en el Alcoià-Comtat frente a comarcas de mayor dinamismo agrarios como el Bajo Segura (cuadro 4). Por su parte, el auge de las granjas de avestruces se relaciona, no tanto con la recepción de ayudas sino sobre todo con la rentabilidad obtenida de estos animales gracias a los elevados precios que se pagan por la carne y los huevos, producción en la que se han especializado las granjas existentes, (10.000 ptas./unidad/huevo y 3.500 ptas./kg./solomillo). Esta renta de posición, sin embargo, podría disiparse si disminuyen los precios al saturarse el mercado; evolución factible si continúa el incremento del número de granjas y, paralelamente, no se amplía la demanda de este producto: sólo se encuentra en ciertos establecimientos de restauración.

\subsection{La difusión de la agricultura ecológica y de nuevos aprovechamientos}

La adopción de técnicas de cultivo compatibles con el medio ambiente es uno de los objetivos de la política agraria comunitaria. Con ella se pretende reducir en parte los desequilibrios generados por unas prácticas intensivas pero también obtener unos productos de calidad, cuya demanda ha experimentado un notable incremento en las últimas décadas. Hacia estas producciones se están encaminando actuaciones tanto públicas, que se han beneficiado de subvenciones procedentes de la Conselleria de Agricultura y del CEDER Aitana, como privadas (agricultores); afectando tanto a productos tradicionales (olivo) como a nuevos aprovechamientos (frutales) e incluso aprovechamientos no considerados como agrícolas (hierbas aromáticas).

Actuaciones que reconocen el hecho de que puede resultar especialmente interesante encaminarse hacia las producciones de calidad y hacia la agricultura integrada, a medio camino entre las prácticas intensivas y las biológicas. Este sistema de cultivo es fácilmente practicable en la zona dadas las técnicas de cultivo tradicionales existentes, mucho más cercanas de lo que los que las practican creen de lo que conocemos como agricultura integrada y que además implicaría escasos costes para su adopción. Especialmente difícil resultaría adoptar la agricultura ecológica en el sentido estricto del término en las áreas más productivas, por lo que quedaría relegada a la recuperación del cultivo en terrazas abandonadas, donde podría resultar especialmente apropiada. Tollos, con el $75 \%$ del término municipal abandonado, podría llegar a ser un municipio especialmente idóneo para el desarrollo de un olivar ecológico, situándose en él las principales iniciativas, todavía en fase de estudio, para la adopción de estas técnicas de cultivo; modelo ya implantado por la cooperativa de Millares (Valencia), donde el $80 \%$ de la superficie destinada al cultivo del olivo está reconocido por el Comité de Agricultura Ecológico de la Comunidad Valenciana.

De las iniciativas que abogan por la extensificación del cultivo del olivo cabe citar la de la cooperativa Blancoana de Alfafara, especialmente dedicada al cultivo de plantas aromáticas ecológicas, que ha puesto en cultivo ecológico una parcela con más de 10.000 olivos, pero que se encuentra con el grave problema de no hallar una almazara donde moler su posible producción con las debidas garantías para obtener un aceite reconocido como ecológico. Esto último resulta paradójico si tenemos en cuenta los graves problemas tenidos por el Ceder Aitana para encontrar parcelas experimentales en su ámbito de actuación para desarrollar su fase experimental del olivar ecológico. También se trabaja desde el Ceder Aitana en la cooperación transnacional con Italia, especialmente orientada hacia una fase experimental del olivo ecológico con parcelas experimentales que se sitúan en Castells, bajo la supervisión de la cooperativa de Sant Isidre y en distintos pueblos 
(Balones, Benillup, Fageca, Planes y Vall d'Alcalá) cuyas cooperativas están integradas en la cooperativa de segundo grado de SOCAPMA (cuadro 2). No obstante, estas prácticas continúan teniendo una escasa relevancia en la comarca. Su justificación es similar a la indicada para el cultivo del olivo mediante técnicas tradicionales: el tradicionalismo y el predominio de la agricultura a tiempo parcial dificulta la introducción de innovaciones. Éstas generalmente las adoptan aquéllos que deseen continuar viviendo de la agricultura conscientes de los mayores beneficios que genera esta práctica, tal y como se ha comprobado en otras regiones europeas (HERNÁNDEZ HERNÁNDEZ, M.: 1999).

La diversificación de rentas se ha encauzado, asimismo, a la introducción de aprovechamientos como las flores y los cultivos tropicales, en los términos municipales de Penáguila y Tárbena, respectivamente (cuadro 2). Estos cultivos podrían considerarse como algo exótico, carentes de sentido en un área interior. Sin embargo, ambos deben entenderse como un complemento de rentas de los aprovechamientos tradicionales, que son mayoritarios en ambas explotaciones; objetivo similar a la difusión de mangos, aguacates y maracuyás en Callosa d'en Sarrià; municipio pionero en la provincia de Alicante en esta práctica. Igualmente se apuesta por aspectos como la calidad frente a la cantidad (los aguacates, por ejemplo, están acogidos a la denominación de agricultura ecológica), la necesidad de controlar la comercialización (la explotación de las flores tiene asegurada la venta de su producción al abastecer al tanatorio de Alcoy) o el propio sistema de explotación, basado en la explotación directa y sobre todo familiar, lo que genera, gracias a esa diversificación de las rentas, empleos estables para unos trabajadores que desean permanecer en este espacio y vivir de la agricultura. No obstante, tampoco debemos olvidar mencionar ciertas dificultades para su adopción y expansión (falta de conocimientos técnicos) o en su evolución futura: monodependencia de ciertos compradores. Además, en el caso concreto de las flores, la importancia de unas temperaturas no muy elevadas en ciertas fases de su cultivo justifica la idoneidad del clima del interior de la provincia; razón que justifica la implantación de una gran explotación ilicitana (Elig flor) en el municipio de Castalla, donde se han puesto en cultivo ocho hectáreas mediante invernaderos dedicadas al cultivo de la rosa variedad gala.

\subsection{Sistemas de gestión de la tierra}

Relevantes han sido las mejoras adoptadas en los sistemas de gestión de las tierras y la creación de empleos estables. Existe una intención clara de incentivar la creación de los bancos de tierra en todos los municipios del área LEADER. La cooperativa Sant Isidre de Castell de Castells y el ayuntamiento de Tollos son pioneros en esta iniciativa. Se trata de una «concentración parcelaria» gestionada por la propia cooperativa con los siguientes objetivos básicos:

- encontrar un cultivo idóneo para cada parcela ya que los medios montanos se caracterizan por una gran variedad de microclimas y suelos,

- buscar solución tanto al abandono de tierras por falta de continuidad en la explotación por parte de los posibles herederos de la misma, imposibilitados o desinteresados en seguir explotando la herencia de sus antecesores, como a poder ampliar la superficie de cultivo de aquellos activos agrarios que quieran seguir ligados a la agricultura,

- generar empleos especializados en determinadas tareas agrícolas (poda, arado, recolección, etc.) de manera que se asegure una complementariedad de rentas de estos especialistas (pluriactividad) al realizarlas en aquellas explotaciones cuyos propietarios carezcan de conocimientos para su realización, 
- racionalizar el uso de la maquinaria agrícola ya que la propia cooperativa adquiere la maquinaria necesaria para todas las tierras que queden bajo su control y se evita así la frecuente infrautilización de la misma.

Las formas de contrato tienen una flexibilidad máxima para repartir gastos, ingresos y labores agrícolas entre los propietarios de la parcela y la cooperativa aunque lo idóneo para la cooperativa es la cesión total de la explotación, no de la propiedad, que permite una mayor eficacia en las labores a llevar a cabo por la misma.

Estas actividades, unidas a los salarios obtenidos mediante el PAMER (limpieza forestal, mantenimiento de caminos rurales, etc.) y el cultivo de sus explotaciones permitirían completar las rentas de estos agricultores. Nos encontraríamos ante un nuevo modelo de pluriactividad, basada en la práctica de diversas tareas relacionadas con el medio rural diferente a la tradicional alternancia entre el trabajo en la propia explotación y el empleo como asalariado en la industria de los núcleos cercanos.

\section{Conclusiones}

La potenciación de los aprovechamientos tradicionales así como la de nuevos usos se fundamentan en una cuestión primordial como es ofrecer una calidad, cada vez más demandada por el mercado frente a una cantidad, que ofrecen las tierras donde la intensificación es más factible. Asimismo, el mantener las actividades agrarias es un elemento fundamental para conservar los paisajes, concretamente, los aterrazamientos, ya que sin ellos la zona perdería uno de los principales atractivos para los turistas, además de generarse elevados procesos de degradación ambiental; pero el problema está en hacer ver a corto plazo el valor de esas externalidades y convertirlas en un bien con mercado. Estas afirmaciones son recogidas por la nueva política agraria comunitaria donde se habla del doble papel del agricultor como productor pero también como protector de la naturaleza. Política un tanto ambigua ya que presenta un tratamiento diferenciado; generalmente se aplica en los países mediterráneos y dentro de ellos en áreas marginales con escasa vocación agrícola donde la comisión «incentiva a los agricultores a que adopten actividades de tutela ambiental mediante la reconversión agroforestal o la adopción de técnicas compatibles con el medio ambiente» (HERNÁNDEZ HERNÁNDEZ, M.; 1999, pág. 56) mientras que éstas son prácticamente testimoniales en las áreas de elevada vocación agrícola.

Sin embargo, este tipo de medidas es difícil de llevar a cabo dado el tradicionalismo de muchos de los habitantes de los medios rurales (envejecimiento, reticencias a la hora de adoptar innovaciones tal y como se ha observado en algunas cooperativas de la zona), pero también dado el escaso beneficio que aportan a corto plazo. Las actuaciones diversificadoras de las actividades económicas en el medio rural no justifican «un cierto antiagrarismo (más real que teórico) en las políticas de desarrollo rural, donde raramente se plantean el análisis del potencial de los sistemas agrarios y las actividades que se priorizan e introducen suelen ser, básicamente, terciarias, ligadas al turismo» (CALATRAVA REQUENA, J.; 1997, pág. 55).

El agricultor y en general el habitante tradicional del medio rural se siente minusvalorado por el urbanita. Uno de los objetivos del desarrollo rural debería ser concienciarlo de lo contrario ya que no se obtendrán resultados positivos de este tipo de políticas, si la población afectada no está convencida de su potencial. En este sentido es muy importante el efecto demostrativo que ciertas actuaciones puedan tener en estos ámbitos. Los ejemplos citados de pluriactividad rural en los que se combinan los empleos generados por el PAMER, el turismo rural, los trabajos agrícolas especializados realizados en los bancos de tierras y las labores desarrolladas en la propia explotación pueden llegar a cumplir ese 
papel. Hay que sensibilizar, asimismo, a la población local de la calidad de vida que oferta «este medio rural». Cabe señalar las posibilidades que ofrecen las nuevas tecnologías (teletrabajo, internet) para un nuevo tipo de población (neorrurales) en este medio rural, donde las comunicaciones están garantizadas en gran parte gracias a la existencia de segundas residencias en urbanizaciones y pueblos. Todas estas actividades innovadoras (teletrabajo, banco de tierras, turismo rural y agricultura ecológica) son adoptadas en un elevado porcentaje por extranjeros. Esta mayor predisposición viene determinada por el hecho de que estos neorrurales, muchos de ellos pensionistas, pueden obtener grandes beneficios (especialmente en calidad de vida), sin arriesgar sus posesiones, como les puede suceder a los habitantes tradicionales.

Es un error, por último, considerar a la Montaña de Alicante como un medio rural homogéneo que requiere soluciones únicas. Cada una de las realidades descritas en epígrafes anteriores requiere su propia intervención. En las tierras incultas o en proceso de abandono, la introducción de técnicas ecológicas permitiría la recuperación de parcelarios sin necesidad de grandes inversiones, además de generar unos ingresos mayores por unidad de superficie; teniendo asegurada su comercialización gracias a la demanda existente, que supera a la producción. La agricultura integrada, por su parte, puede resultar especialmente apropiada en aquellas zonas extensivas tradicionales, dominantes en esta comarca dada la orografía existente. Para las explotaciones de tipo medio-grandes, cuyos titulares son generalmente agricultores a título principal, la intensificación y la tecnificación es la opción más socorrida, con el claro objetivo de reducir costes, especialmente los salariales, e incrementar los beneficios para mantener la plena dedicación, que en los casos anteriores sería posible mediante la pluriactividad.

\section{Bibliografía}

CALATRAVA REQUENA, J. (1997): «El olivar en los procesos de desarrollo rural: consideraciones sobre su valor económico global», en La Reforma de la OCM y el futuro del olivar, Universidad de Jaén, Jaén, pp. 53-76.

GENERALITAT VALENCIANA (1994): Programa Agroalimentario de la Comunidad Valenciano (1994-2000), Conselleria de Agricultura, Pesca y Alimentación, 326 pp.

HERNÁNDEZ HERNÁNDEZ, M. (1996): «Las Políticas de intervención en la Montaña de Alicante: repercusiones sobre los paisajes agrarios tradicionales», en Montagnes Mediterraneennes, $n^{o}$ 4, Conseil General de l'Ardeche, Association RGA, Universitè Joseph Fourier, Grenoble, (Francia), pp. 109-112.

- (1999): «Las repercusiones espaciales de las políticas ambientales en el Veneto (Italia)», en Investigaciones Geográficas, $n^{\circ}$ 22, Instituto Universitario de Geografía, Universidad de Alicante, pp. 53-68.

- (en prensa): «La Agricultura y la conservación del medio ambiente», en Eines per al desenvolupament local, Universidad de Alicante-Ceder Aitana, Cocentaina.

HERNÁNDEZ HERNÁNDEZ, M. y MOLTÓ MANTERO, E. (en prensa): «Intervenciones en el espacio agrario de la Montaña de Alicante», en Actas XVI Congreso de Geógrafos Españoles «El territorio y su imagen, Asociación de Geógrafos Españoles-Departamento de Geografía de la Universidad de Málaga.

MOLTÓ MANTERO, E. (1998): «La agricultura tiempo parcial en 'La Montaña'» (Alicante), IX Coloquio de Geografía Rural, Asociación de Geógrafos Españoles-Universidad del País Vasco, Vitoria, pp. 139-146.

PÉREZ PÉREZ, D. (1997): Restructuració dels espais industrials de l'eix Alcoi-Cocentaina-Muro, Instituto de Cultura Juan Gil-Albert, Diputación Provincial de Alicante, Alicante.

RODRIGO ALFONSO, C. (1999): «Un leader trencaclosques», en Ruralia, 3, Centro Rural de información Europea (C.R.I.E), Castellón. 